\title{
Preliminary investigation evaluating college students' willingness and need to take pre-exposure prophylaxis (PrEP) for HIV
}

\author{
Trisha Arnold ${ }^{1}$, Courtney E. Sims-Gomillia ${ }^{2}$, Kaitlin Portz ${ }^{3}$, Estefany Bologna ${ }^{3}$, Bryman Williams ${ }^{3}$ \\ ${ }^{1}$ Brown Unviersity, United States \\ ${ }^{2}$ University of Mississippi Medical Center, United States \\ ${ }^{3}$ Jackson State University, United States
}

\begin{abstract}
Pre-exposure prophylaxis (PrEP) is a highly effective pharmacological approach to HIV prevention. This study sought to evaluate the need and willingness of African American college students to utilize PrEP. We evaluated students' perceived HIV risk, beliefs about medication, sexual risk behaviors, and need for PrEP. The sample consisted of 147 participants $\left(\mathrm{M}_{\text {age }}=21.6\right.$ years, $\left.\mathrm{SD}_{\text {age }}=4.8\right)$, and $57.7 \%$ were female. Most of the sample had been sexually active in the past. Participants were willing to initiate PrEP if they knew it would lower their chance of contracting HIV (88.2\%), knew their partner was HIV positive (85.4\%), or thought they were at high-risk for HIV infection (87.9\%). Additionally, there was a strong positive relationship between willingness to initiate PrEP and beliefs about medicine $(r=0.217, p<0.05)$. These findings have relevant implications that will aid in the promotion of PrEP uptake and initiation among at-risk college students.
\end{abstract}

HIV AIDS Rev 2018; 17, 2: 98-102

DOI: https://doi.org/10.5114/hivar.2018.76370

Key words: pre-exposure prophylaxis (PrEP), college students, HIV, sexual risk behaviors, beliefs about medication, perceived risk.

\section{Introduction}

It is estimated that 1.1 million people currently live with HIV/AIDS in the United States (U.S.) [1]. Between 2009 and 2013, there were 232,003 new human immunodeficiency virus (HIV) diagnoses in the U.S. [2]. Deaths caused by HIV-related illnesses increased by $50 \%$ among young populations from 2005 to 2012 [3]. African Americans are at a higher risk of acquiring HIV, with $46 \%$ of all new infections between 2009 and 2013 occurring in this population, and a disproportionate number of these new infections are occurring in Southern states [2]. In particular, Mississippi (MS) has the $9^{\text {th }}$ highest rate of new HIV diagnoses, and Jackson (the study site) ranks $8^{\text {th }}$ in overall prevalence of HIV cases nationwide [2]. Jackson also has the fastest rate of new HIV infections in the US among men who have sex with men (MSM) [4]. Given the disproportionate burden of HIV in Mississippi and among African Americans, innovative and targeted HIV prevention strategies must be developed.

Pre-exposure prophylaxis (PrEP) is an innovative pharmacological approach developed to prevent HIV transmission. PrEP has demonstrated up to $92 \%$ efficiency in reducing transmission of HIV infection [5]. Although PrEP has proven clinical efficacy, it has yet to be fully utilized by prescribing providers. Research has demonstrated that

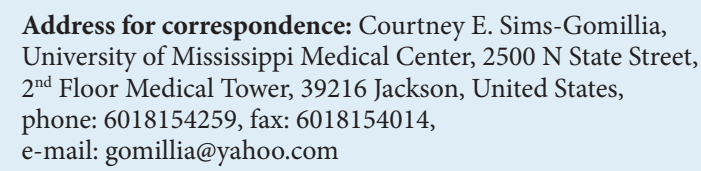

Article history:

Received: 10.08.2017

Received in revised form: 14.11.2017

Accepted: 10.01.2018

Available online: 21.05.2018
International Journal of HIV-Related Problems

HIV \& AIDS

R e vi e w 
there is low PrEP awareness and low prescribing of PrEP, while the overall interest in PrEP is high [6-9]. In addition, barriers that prevent the effective optimization and implementation of PrEP still exist [10-12]. These barriers include several psychosocial, social, and structural factors that affect the implementation of PrEP among individuals that could otherwise benefit [7].

Although PrEP is an effective strategy to reduce the risk of HIV infection, the likelihood of its use remains unknown. To our knowledge, few studies have been designed to specifically examine the need and willingness of at risk college students to take PrEP $[13,14]$. The scarcity of research regarding acceptance and willingness to take PrEP in young college populations at risk in the U.S. warrants the need for further study. Therefore, the present study evaluated the attitudes about and willingness to take PrEP within a sample of college students attending a Historically Black College/University (HBCU) in the Southern U.S. Information relevant to students' perceived HIV risk, beliefs about medication, sexually risky behaviors, perceived need for PrEP medication, and a series of demographic characteristics were collected. We also assessed the need for PrEP according to self-reported risk behaviors and willingness to take PrEP. In the current study, we hypothesized: 1) individuals who are clinically recommended to take PrEP will score high on willingness to initiate PrEP and high on perceived risk for HIV; 2) there will be a strong positive relationship between willingness to initiate PrEP and perceived risk of HIV score; 3 ) there will be a strong positive relationship between willingness to initiate PrEP and beliefs about medicine.

\section{Material and methods}

\section{Participant demographics}

A total of 147 participants were recruited from undergraduate introductory courses to participate in this study. There was rather equal distribution of gender among the sample, with women representing just over half (57.7\%) of the sample. The mean age was 21.6 years $(\mathrm{SD}=4.8)$. Participants were mostly African American (89.1\%). Most $(89.8 \%)$ of the sample identified as heterosexual, nearly $7 \%$ were bisexual, and 3.4\% identified as gay or lesbian. All college classifications were represented in this sample: $14.3 \%$ freshmen, $30.6 \%$ sophomores, $28.6 \%$ juniors, and $29.5 \%$ seniors. Nearly $91 \%$ of the sample were single and had never been married. About half (56.5\%) of participants lived on campus and most (85.7\%) had health insurance. A summary of the demographic characteristics is presented in Table 1.

\section{Data collection}

Questionnaires were administered via paper and pencil. A general demographic questionnaire was used to collect age, gender, geographic information, ethnicity, college classification, annual household income, and number of people living in the home, among other questions. Participants
Table 1. Demographic characteristics of sample $(N=147)$

\begin{tabular}{|c|c|}
\hline Demographic characteristics & $\%$ of sample \\
\hline \multicolumn{2}{|l|}{ Gender } \\
\hline Women & 57.8 \\
\hline Men & 42.2 \\
\hline \multicolumn{2}{|l|}{ Age } \\
\hline Total sample & $21.6 \pm 4.8$ \\
\hline \multicolumn{2}{|l|}{ Ethnicity } \\
\hline African American/Black & 89.1 \\
\hline Biracial/Multiracial & 4.1 \\
\hline American Indian/Alaskan native & 3.1 \\
\hline White & 3.4 \\
\hline Hispanic & 0.7 \\
\hline \multicolumn{2}{|l|}{ Religion } \\
\hline Baptist & 59.9 \\
\hline Methodist & 4.8 \\
\hline Catholic & 4.1 \\
\hline Agnostic & 2.1 \\
\hline Non-denominational & 9.6 \\
\hline Church of Christ & 2.2 \\
\hline Other or no response & 17.3 \\
\hline \multicolumn{2}{|l|}{ Sexual orientation } \\
\hline Heterosexual & 89.8 \\
\hline Bisexual & 6.8 \\
\hline Gay/lesbian & 3.4 \\
\hline \multicolumn{2}{|l|}{ College classification } \\
\hline Freshman & 14.3 \\
\hline Sophomore & 30.6 \\
\hline Junior & 28.6 \\
\hline Senior & 29.5 \\
\hline
\end{tabular}

were asked about their awareness of PrEP and were provided a brief written description of PrEP. Willingness to use PrEP was measured using a dichotomous 'Yes' or 'No' question. The Beliefs about Medication Questionnaire was administered to evaluate students' beliefs about medication [15]. This measure has reliability within acceptable limits at $\alpha=0.78$ and criterion validity at 0.23 . High scores on this measure suggest that a person does not believe medication helps manage illnesses. The Perceived Risk of HIV Scale was used to assess the participants' perceived risk of contracting HIV [16]. Overall, the PRHS has good reliability of $\alpha=0.85$ and concurrent criterion-related validity of 0.88 . Currently, there is no validated measure to assess a person's need for PrEP, thus questions related to participants' need for a PrEP prescription were developed for the purpose of this study. These questions were based on research by Project Inform, which identified risk behaviors that may qualify 
Table 2. Questions assessing need for pre-exposure prophylaxis

Please Circle One Answer for Each Question.

\begin{tabular}{|c|c|c|}
\hline In the last 6 months did you know or suspect any of your partner(s) to have HIV? & Yes & No \\
\hline Have you had sex within the past 6 months without using condoms? & Yes & No \\
\hline In the last 6 months did any of your partner(s) refuse to use condoms with you? & Yes & No \\
\hline Are you African American or Latino/a? & Yes & No \\
\hline In the last 6 months did you have more than one sex partner, even if it was only every once in a while? & Yes & No \\
\hline In the last 6 months did your partner(s) ever have other sex partners, or do you suspect he/she does? & Yes & No \\
\hline $\begin{array}{l}\text { In the last } 6 \text { months were any of your partner(s) treated for an STD, such as syphilis, gonorrhea, herpes, } \\
\text { or chlamydia? }\end{array}$ & Yes & No \\
\hline In the last 6 months did you experience sexual or other types of violence in your life? & Yes & No \\
\hline In the last 6 months did you ever feel you didn't have control or were not able to use a condom during sex? & Yes & No \\
\hline In the last 6 months did any of your partner(s) inject drugs or did they ever use injection drugs? & Yes & No \\
\hline In the last 6 months your partner(s) ever been in jail or prison? & Yes & No \\
\hline In the last 6 months were you affected by depression, low self-esteem or anxiety? & Yes & No \\
\hline $\begin{array}{l}\text { In the last } 6 \text { months have you or your partner(s) ever exchanged sex for money, drugs, housing, or other } \\
\text { kinds of assistance? }\end{array}$ & Yes & No \\
\hline In the last 6 months did you inject drugs with needles? & Yes & No \\
\hline In the last 6 months did you have sex after drinking alcohol or using recreational drugs? & Yes & No \\
\hline Do you know your current HIV status? & Yes & No \\
\hline When was the last time you were tested for HIV? & Year: & \\
\hline Are you in regular medical care? & Yes & No \\
\hline Do you have health insurance? & Yes & No \\
\hline
\end{tabular}

a person to receive a PrEP prescription (see Table 2) [17]. The principal investigator collaborated with Project Inform team members, who reviewed our newly created measures and made suggestions that were then incorporated. If participants endorsed six or more of the risk items, or endorsed the question asking if they thought any of their partners in the last six months had HIV, they qualified for 'needing' a PrEP prescription.

\section{Statistical analysis}

For hypothesis one: individuals who meet the criteria to initiate PrEP will score high on willingness to initiate PrEP and high on perceived risk for HIV, a 2 x 2 Between-Subjects MANOVA was performed to determine how individuals who met the criteria to initiate PrEP would score on willingness to initiate PrEP and on perceived risk of HIV. For hypothesis two: there will be a strong positive relationship between willingness to initiate PrEP and perceived risk of HIV score, a bivariate Pearson's correlation coefficient $r$ was completed to evaluate the relationship between willingness to initiate PrEP and perceived risk for HIV. For hypothesis three: there will be a strong positive relationship between willingness to initiate PrEP and beliefs about medicine, a bivariate Pearson's $r$ was completed to evaluate the relationship between willingness to initiate PrEP and beliefs about medicine.

\section{Results}

\section{Descriptive statistics}

Most (87.7\%) of the sample had been sexually active, and a total of $19.7 \%$ of the sample met the 'at-risk' criteria of contracting HIV given our specified study criteria. However, only $10 \%$ of our sample had previously heard about PrEP. When participants were asked if they were willing to initiate PrEP, just over half (53.1\%) reported being willing to initiate PrEP if medication was offered. When participants were asked to rate their willingness to initiate $\operatorname{PrEP}(1=$ 'not willing to take PrEP' to 5 = 'completely willing to take PrEP'), many were willing to initiate $(\mathrm{M}=3.1 ; \mathrm{SD}=1.3)$. In addition, most participants were willing to initiate PrEP if they knew it could lower their chance of acquiring HIV (88.2\%), knew their partner was HIV positive (85.4\%), or thought they were at high risk for HIV infection (87.9\%).

Only $4.1 \%$ of our sample reported currently living with HIV. However, our results may reflect an underreporting of HIV status given that many participants (28.6\%) failed to report when their last HIV test was performed, and only $50.3 \%$ reported being tested within the last year. In addition, less than a quarter $(17.7 \%)$ of participants reported being tested 1 to 2 years prior to data collection. Very few participants (3.4\%) reported that they had never been tested for HIV. Most participants reported having some concerns 
about initiating PrEP, with only $7.9 \%$ reporting no concerns. Side effects associated with the medication were reported as a worry by the majority (89.3\%) of participants. Paying for the medication was also commonly reported as a barrier to initiating PrEP (64.3\%). Fewer participants were worried about the interactions PrEP may have with alcohol (37.9\%) or other medications (34.3\%).

\section{Inferential statistics}

No statistically significant results for hypothesis one were found, which indicates that the individuals who met the criteria to initiate PrEP did not score high on willingness to initiate PrEP and on perceived risk for $\operatorname{HIV} F(1,146)=11.72$, $p=0.718$. For hypothesis two, we also found no positive relationship between willingness to initiate PrEP and perceived risk of HIV. Finally, concerning hypothesis three, we found a positive relationship between willingness to initiate $\mathrm{PrEP}$ and beliefs about medicine $(r=0.217, p<0.05)$.

\section{Discussion}

In this sample of primarily African American students attending a HCBU in the South, a number of interesting outcomes about willingness to initiate PrEP and possible influences on PrEP initiation were present. We expected to find that individuals who met criteria to start PrEP would score higher on perceived risk (hypothesis one), and that there would be a strong positive relationship between willingness to initiate PrEP and the perceived risk of HIV (hypothesis two), but our results did not support these hypotheses. However, we did find that there was a strong positive relationship between willingness to initiate PrEP and beliefs about medicine (hypothesis three).

The results from the current study differ in some ways from previously published literature on young adults at risk of acquiring HIV [18]. For example, high acceptance and willingness to initiate PrEP after being educated about PrEP has been previously found [18]. Therefore, our findings are particularly noteworthy given the high rates of HIV in the South, and may indicate a lack of awareness of HIV risk in individuals who would benefit from taking PrEP. In the current study, only $10 \%$ of the sample had previously heard about PrEP, displaying a clear lack of PrEP knowledge, which may explain why those at perceived higher risk were not more willing to take PrEP.

Some of our results are similar to findings in previous literature in that participants were willing to initiate PrEP if they thought they were at risk of acquiring HIV and were overall somewhat accepting of PrEP medication [13, 14, 18-20]. Similar to other studies, most participants reported some concerns with initiating PrEP [21]. Potential side effects were the most reported concern with PrEP initiation. This study also found that the cost of the medication was also a common concern. Interestingly, a small number of students were concerned with the interaction that the medica- tion may have with alcohol. For persons prescribed PrEP, it is essential that they not only become educated on side effects and proper medication use but also on other pertinent information, such as drug interactions.

Our results point to the need to provide more targeted education about both HIV risk and PrEP's potential as an alternate HIV prevention method. Specifically, there should be educational campaigns aimed toward young students attending HCBUs about HIV risk factors, PrEP, and the existence of PrEP patient assistance programs that can supplement the cost of the medication. These awareness and educational efforts could be incorporated into existing HIV prevention efforts such as National Black HIV/AIDS Awareness Day events [21].

\section{Limitations and future research}

The current study has three notable limitations. First, the data were collected using a convenience sample of college students. Thus, sample bias could be present due to data collection solely with available college students. A clinical sample may better represent young adults with high-risk levels for HIV. Second, due to the lack of a standardized measure to determining if a person should initiate PrEP, we created our own questionnaire. We determined the threshold to be considered 'at-risk' to be six risk items. This number was selected based on CDC recommendations for providers prescribing PrEP, yet it may not be the most accurate way to ascertain who would benefit from taking PrEP, since prescribing PrEP may require a more individual risk-based assessment. Third, the data were collected in classes, while participants were surrounded by their peers, which could have caused some reluctance to answer sensitive questions about sexual risk behavior. This study would have benefited from adding a social desirability scale to understand if the environment, in which the questionnaire was administered influenced participants' response styles.

\section{Conclusions}

Assessing the need and willingness of African American college students to utilize PrEP offers great insight in regard to PrEP acceptance. Based on our findings that suggested little awareness of PrEP and minimal concerns of its use, there is a need for additional education on PrEP as an HIV prevention method. Knowing students' perceived HIV risk, beliefs about medication, sexually risky behaviors, and need for PrEP medication offers important implications that will aid in the promotion of PrEP uptake and initiation among at-risk college students.

\section{Conflict of interest}

The authors declare no potential conflicts of interest with respect to the research, authorship, and/or publication of this article. 


\section{References}

1. Prejean J, Song R, Hernandez A, et al.; HIV Incidence Surveillance Group. Estimated HIV incidence in the United States, 2006-2009. PLoS One 2011; 6: e17502.

2. Centers for Disease Control and Prevention (2015). Diagnoses of HIV Infection in the United States and Dependent Areas, 2013. Retrieved from: http://www.cdc.gov/hiv/library/reports/surveillance/

3. UNAIDS report on the global AIDS epidemic. WHO Library Cataloguing-in-Publication Data, Geneva 2013.

4. Rosenberg ES, Grey JA, Sanchez TH, et al. Rates of Prevalent HIV Infection, Prevalent Diagnoses, and New Diagnoses Among Men Who Have Sex with Men in US States, Metropolitan Statistical Areas, and Counties, 2012-2013. JMIR Public Health Surveill 2016; 2: e22.

5. Underhill K, Morrow KM, Colleran C, et al. Explaining the Efficacy of Pre-Exposure Prophylaxis (PrEP) for HIV Prevention: A Qualitative Study of Message Framing and Messaging Preferences among US Men Who Have Sex with Men. AIDS Behav 2016; 20: 15141526.

6. Cohen SE, Vittinghoff E, Bacon O, et al. High interest in preexposure prophylaxis among men who have sex with men at risk for HIV infection: baseline data from the US PrEP demonstration project. J Acquir Immune Defic Syndr 2015; 68: 439-448.

7. Oldenburg CE, Perez-Brumer AG, Hatzenbuehler ML, et al. Statelevel structural sexual stigma and HIV prevention in a national online sample of HIV-uninfected MSM in the United States. AIDS 2015; 29: 837-845.

8. Van der Elst EM, Mbogua J, Operario D, et al. High acceptability of HIV pre-exposure prophylaxis but challenges in adherence and use: qualitative insights from a phase I trial of intermittent and daily PrEP in at-risk populations in Kenya. AIDS Behav 2013; 17: 2162-2172.

9. Bauermeister JA, Meanley S, Pingel E, et al. PrEP awareness and perceived barriers among single young men who have sex with men. Curr HIV Res 2013; 11: 520-527.

10. Golub SA, Gamarel KE, Rendina HJ, et al. From efficacy to effectiveness: facilitators and barriers to PrEP acceptability and motivations for adherence among MSM and transgender women in New York City. AIDS Patient Care STDS 2013; 27: 248-254.

11. Krakower DS, Mimiaga MJ, Rosenberger JG, et al. Limited Awareness and Low Immediate Uptake of Pre-Exposure Prophylaxis among Men Who Have Sex with Men Using an Internet Social Networking Site. PLoS One 2012; 7: e33119.

12. Rucinski KB, Mensah NP, Sepkowitz KA, et al. Knowledge and use of pre-exposure prophylaxis among an online sample of young men who have sex with men in New York City. AIDS Behav 2013; 17: 2180-2184.

13. Whiteside YO, Harris T, Scanlon C, et al. Self-perceived risk of HIV infection and attitudes about preexposure prophylaxis among sexually transmitted disease clinic attendees in South Carolina. AIDS Patient Care STDS 2011; 25: 365-370.

14. Young I, McDaid L. How acceptable are antiretrovirals for the prevention of sexually transmitted HIV?: A review of research on the acceptability of oral pre-exposure prophylaxis and treatment as prevention. AIDS Behav 2014; 18: 195-216.

15. Horne R, Graupner L, Frost S, et al. Medicine in a multi-cultural society: the effect of cultural background on beliefs about medications. Soc Sci Med 2004; 59: 1307-1313.

16. Napper LE, Fisher DG, Reynolds GL. Development of the perceived risk of HIV scale. AIDS Behav 2012; 16: 1075-1083.

17. PrEP a new option for women for safer loving. A Publication for Women Who Sleep with Men. Project Inform, San Francisco 2014.

18. Smith DK, Toledo L, Smith DJ, et al. Attitudes and program preferences of African-American urban young adults about pre-exposure prophylaxis (PrEP). AIDS Educ Prev 2012; 24: 408-421.
19. Mustanski B, Johnson AK, Garofalo R, et al. Perceived likelihood of using HIV pre-exposure prophylaxis medications among young men who have sex with men. AIDS Behav 2013; 17: 2173-2179.

20. Galea JT, Kinsler JJ, Salazar X, et al. Acceptability of pre-exposure prophylaxis as an HIV prevention strategy: barriers and facilitators to pre-exposure prophylaxis uptake among at-risk Peruvian populations. Int J STD AIDS 2011; 22: 256-262.

21. The National Black HIV/AIDS Awareness Day HBCU Initiative, 2016. Retrieved from: https://nationalblackaidsday.org/hbcu-initiative/ 